\title{
Highly-Integrated Quad-Channel Transimpedance Amplifier for Next Generation Coherent Optical Receivers
}

\author{
Rajanish Pandey ${ }^{1}$, Greg Takahashi², Shanthi Bhagavatheeswaran ${ }^{1}$, Eric Tangen ${ }^{1}$, \\ Matt Heins ${ }^{1}$, and Jens Muellrich ${ }^{3}$ \\ 1DP Group, Qorvo, Richardson, TX, 75080, USA, rajanish.pandey@ qorvo.com, \\ shanthi.bhagavatheeswaran@qorvo.com, eric.tangen@qorvo.com, matt.heins@qorvo.com \\ ${ }^{2}$ IDP Group, Qorvo, SJ, CA, 95134, USA, greg.takahashi@qorvo.com \\ ${ }^{3}$ Micram Microelectronic GmbH, Bochum, Germany, jens.muellrich@micram.com
}

\begin{abstract}
This paper presents a highly integrated, high performance four channel linear transimpedance amplifier (TIA) RFIC with a footprint of $2 \mathrm{~mm} \times 3.5 \mathrm{~mm}$ towards next generation $100 \mathrm{G} / 400 \mathrm{G}$ miniaturized coherent receivers. A TIA of such form may become indispensable as the size, complexity and cost of receivers continue to reduce. The design has been realized in a $130 \mathrm{~nm}$ SiGe BiCMOS process for a low cost, high performance solution towards longhaul/metro applications. The TIA is capable of providing control functions either digitally through an on-chip 4-wire serial-peripheral interface (SPI) or in analog mode. Analog mode is provided as an alternative control for real-time control and monitoring. To provide high input dynamic range, a variable gain control block is integrated for each channel, which can be used in automatic or manual mode. The TIA has a differential input, differential output configuration that exhibits state-of-the-art THD of $<0.9 \%$ up to $500 \mathrm{mV}_{\mathrm{pp}}$ output voltage swing for input currents up to $2 \mathrm{~mA}_{\mathrm{pp}}$ and high isolation $>40 \mathrm{~dB}$ between adjacent channels. A high transimpedance gain $(\mathrm{Zt})$ up to $\sim 7 \mathrm{~K} \Omega$ with a large dynamic range up to $37 \mathrm{~dB}$ and variable bandwidth up to $34 \mathrm{GHz}$ together with low average input noise density of $20 \mathrm{pA} / \sqrt{ } \mathrm{Hz}$ has been achieved. To the authors' knowledge, these metrics combined with diverse functionality and high integration have not been exhibited so far. This paper intends to report a state-of-the-art high-baud rate TIA and provide insight into possibilities for further integration.
\end{abstract}

Index Terms - Transimpedance amplifier, coherent receiver, long-haul, photo-diode, SPI, SiGe, micro-ICR.

\section{INTRODUCTION}

Global network traffic driven by $3 \mathrm{G} / 4 \mathrm{G}$ mobile data, data centers and cloud services are driving the demand for high linearity low cost products for the long haul/metro optical transport market. Long haul optical systems at $100 / 400 \mathrm{~Gb} / \mathrm{s}$ use highly efficient modulation schemes such as M-ary phase shift keying and quadrature amplitude modulations, and compact receiver modules towards low cost, highly efficient solutions [1]. The transimpedance amplifier (TIA) forms the key electrical component in integrated coherent receivers (ICR) that receives the photodiode current and amplifies it to an appropriate output voltage with low distortion while adding minimum noise. With form factors of receiver modules scaling down to CFP4, there is an obvious necessity for micro-ICRs. This in turn demands integration of multiple channels of TIAs with associated functions as a single chip solution [2],[3],[4]. At present, manufacturers are using multiple dual channel TIAs with tighter routing and packaging techniques to provide ICR solutions. For example, for $100 \mathrm{~Gb} / \mathrm{s}$ systems, two dualchannel TIA chips are being used. This obviously has higher cost and poses several signal integrity issues in the ICR together with complex routing and poor yield. The TIA presented in this paper provides an efficient solution to overcome such need for the first time.

The block diagram of the TIA presented in this paper is shown in Fig.1. It consists of four TIA channels arranged in parallel as a single chip RFIC. The control and monitoring function of the TIA such as gain control, gain monitoring in auto mode, bandwidth control, peak detect monitoring, photo-diode current monitoring, etc., are controlled by serial peripheral interface (SPI) circuitry embedded in the same chip. As the ICR manufacturers are still evolving with respect to on chip SPI control, a complimentary analog control mode is implemented alongside. This chip has been realized in $130 \mathrm{~nm}$ SiGe BiCMOS process. The TIA has a differential input, differential output configuration that exhibits state-of-theart performance coupled with tight integration and is capable of catering to $32 / 45 \mathrm{~Gb} / \mathrm{s}$ coherent applications. In the following sections we will detail various aspects of the design, operation and measurements of the TIA.

\section{OVERVIEW OF DESIGN AND OPERATION}

The RF chain of the TIA is designed as a broadband fully differential configuration as shown in Fig. 2. It is optimized for the use of balanced PDs and provides high common mode rejection. The input of the TIA is DC coupled to the PDs and also provides anode bias for the 
photodiodes at $1.3 \mathrm{~V}$. The first stage is a shunt feedback, current to voltage converter stage with a tail current source as shown in Fig. 3. The feedback topology provides low noise figure for a given gain-bandwidth product compared to other topologies [5].

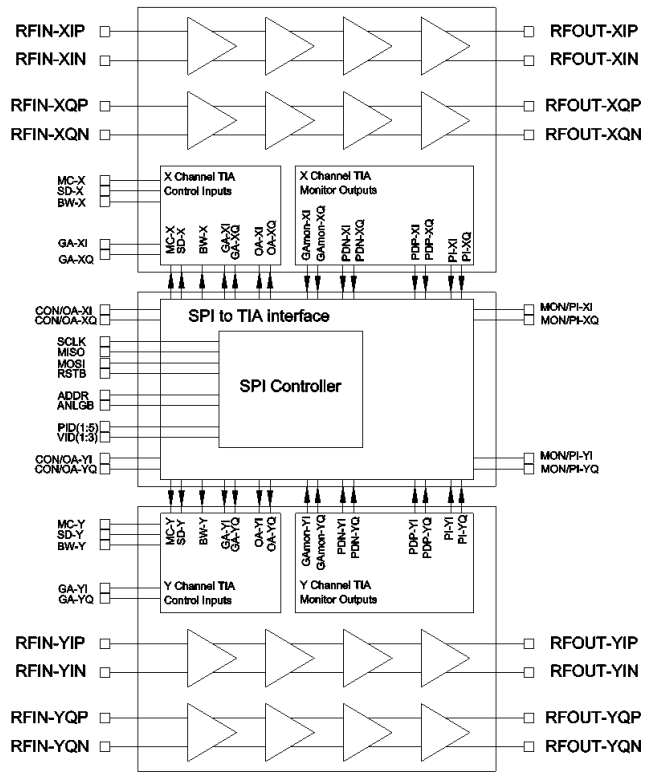

Fig. 1. Block diagram of the complete chip

The subsequent two stages are variable gain amplifier (VGA) stages as shown in Fig. 2, which provide sufficient variable gain dynamic range at a fixed output voltage for a given input dynamic range of signal current. The VGA stages are Gilbert cell types that can provide sufficient dynamic range as well as high isolation between the input and output.

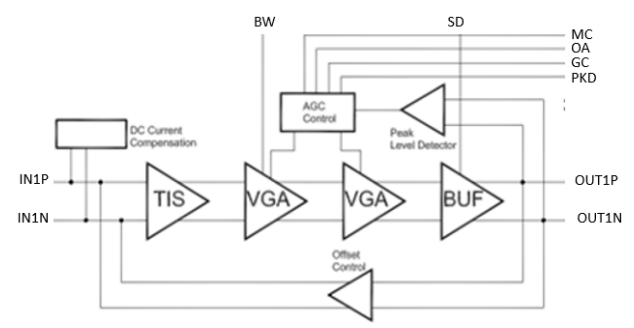

Fig. 2. RF chain lineup of TIA core with placement of gain, offset control, peak detector and DC current compensation

The Gilbert cells also utilize emitter degeneration resistors and capacitors. Emitter degeneration resistors provide the gain conditioning to minimize harmonic distortion of the signal. Degenerating capacitors provide gain boost at higher frequencies to extend the bandwidth set by the first TIA stage. Output buffer stage is a cascode configuration that allows output isolation and very linear output swing up to $900 \mathrm{mV}$ in $100 \Omega$ differential load.

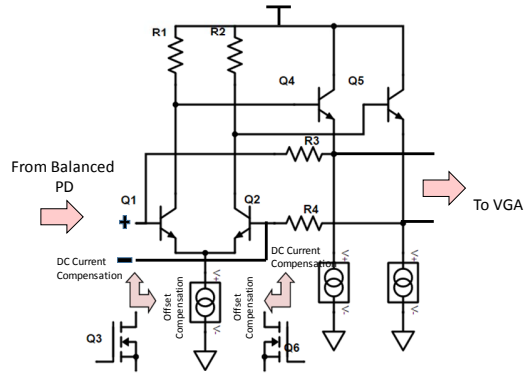

Fig. 3. Shunt Feedback transimpedance stage with DC current and offset compensation strategy

The gain of the VGA stages can be controlled either manually or through an automatic gain control loop. In manual gain control mode (MGC), DC control voltages of up to $3 \mathrm{~V}$ can be applied from bond pads. In automatic gain control mode (AGC), gain is automatically adjusted by a comparator circuit that compares the output voltage generated by a peak detector to a variable reference voltage. Selection of MGC or AGC mode can be achieved digitally through the SPI or manually through bondpads.

The SPI uses a 32 bit serial data interface. In SPI control mode, each TIA channel is controlled separately with a serial data transfer over the SPI interface using serial clock (SCLK), master-out-slave-in (MOSI), masterin-slave-out (MISO) and slave select (SSN) pins. The SPI interface provides control of mode settings (MGC/AGC), individual shutdown of channels and bandwidth control. Bandwidth control is through integrated 4-bit digital-toanalogue converters (DAC).

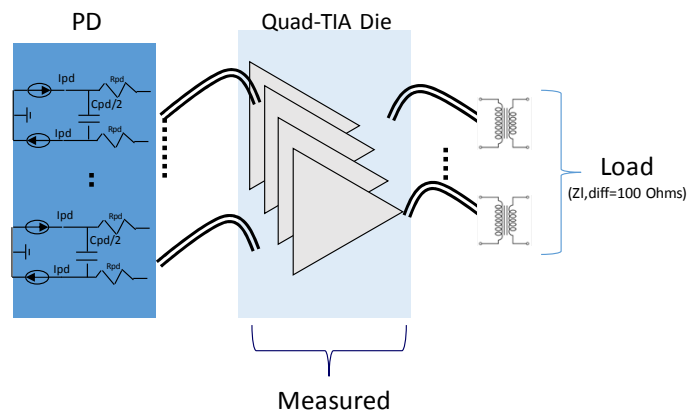

Fig. 4. Methodology to obtain frequency response characteristics of TIA from measured S-parameters

The SPI interface is also used to multiplex different monitor voltages such as the gain monitor voltage, photo diode DC currents and output peak detector voltage through a single pad, hence saving considerable die area. A chip micrograph is shown in Fig 10. The gain monitor is useful when TIA is in automatic gain control mode. Input photo diode current monitors can be used to 
calibrate the optical local oscillator power levels and output peak detectors provide a measure of strength of output voltage.

Since coherent detection can incur large DC currents that flow into the amplifier inputs, DC current monitors at both $\mathrm{p}$ and $\mathrm{n}$ side of differential pair and a DC cancellation circuit that is always active, has been incorporated that compensates for these input currents and stabilizes the input voltage. This can be especially relevant for colorless applications in which multiple optical signals can arrive at a single receiver. An internal offset control loop is also implemented, which senses the offset at the circuit output and applies a differential correction control current to the input of the circuit. This offset control loop compensates not only for circuit asymmetries, but also for asymmetrical input signal strengths (input DC currents).

\section{MEASUREMENT RESULTS}

A comprehensive electrical-input/electrical-output (E/E) measurement was done to characterize the die. RF probes connected to the input and output pads were used for the small signal and linearity measurements. A fourport network analyzer, PNA-X, in true stimulus mode was used for fully differential measurements and a diff-IQ module was used to perform total harmonic distortion (THD) measurements. The S-parameters obtained from these measurements were post processed with PD capacitance of $60 \mathrm{fF}$ and input and output bondwires of $300 \mathrm{pH}$ each to obtain the net frequency response as shown in Fig. 4. The output noise measurement was done using an $\mathrm{N}$-series spectrum analyzer. The output noise is averaged and divided by the gain in order to calculate the average input referred noise.

Typical 3-dB small signal bandwidth measured was 32 $\mathrm{GHz}$ taking into account $\mathrm{PD}$ and bondwires as shown in Fig. 5. This bandwidth can support $32 \mathrm{~Gb} / \mathrm{s}$ and $45 \mathrm{~Gb} / \mathrm{s}$ coherent applications with an appropriate PD. The bandwidth is tunable using the integrated 16-level DAC in the range from $29 \mathrm{GHz}$ to $34 \mathrm{GHz}$. Group delay was measured to be within $+/-10$ ps up to the $3-\mathrm{dB}$ bandwidth.

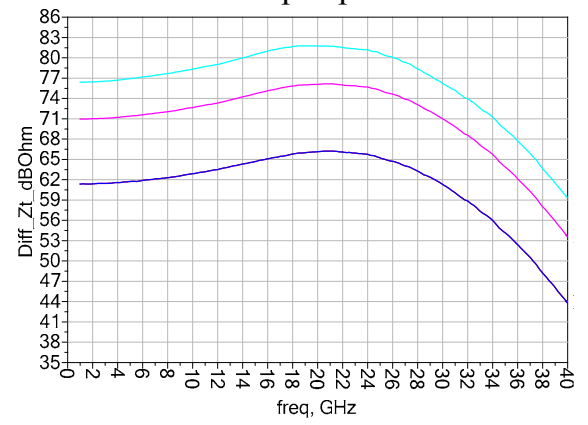

Fig. 5. Measured frequency response of one channel at low (blue), medium (pink) and high gain (sky blue) settings
THD is one of the most critical parameters for future coherent applications. It was measured for a $1 \mathrm{GHz}$ fundamental tone at various input current levels for a fixed output voltage swing. For an output voltage $<=500 \mathrm{mV}_{\mathrm{pp}}$, which is a typical voltage of operation in the final system, state-of-the art voltage THD of $<0.9 \%$, was measured at differential input current levels up to $2 \mathrm{mApp}$ and $<1.2 \%$ up to $3 \mathrm{mApp}$ as shown in Fig. 6. Furthermore, the linearity extends for output voltages up to $900 \mathrm{mVpp}$ and is below $2.5 \%$ up to currents of $3 \mathrm{mApp}$.

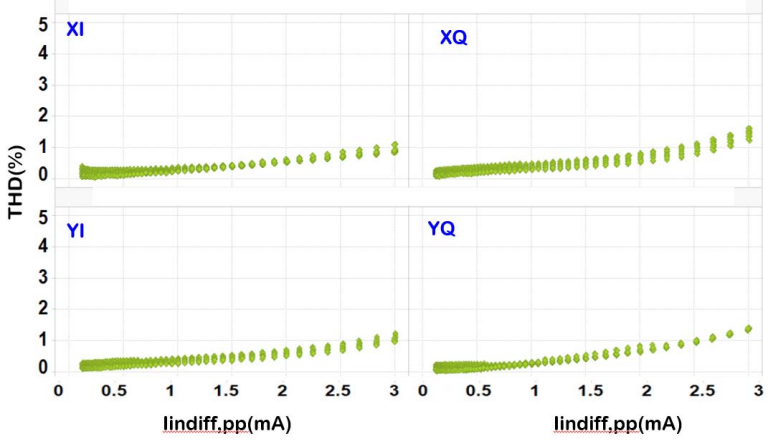

Fig. 6. Measured voltage THD of TIA of all four channels for output voltage of $500 \mathrm{mVpp}$

As shown in Fig. 7, adjacent channel isolation of $>40 \mathrm{~dB}$ was measured between all four channels across the full bandwidth which represents state-of-the-art performance figure for such densely integrated circuit. Average input referred noise (IRN) measured over 3-dB bandwidth was $20 \mathrm{pA} / \mathrm{sqrt}(\mathrm{Hz})$, shown in Fig. 8. Further reduction of IRN may be needed to improve signal to noise ratio $(\mathrm{S} / \mathrm{N})$, for optical receivers using higher level modulation techniques. Since the compensation circuits contribute about $20 \%$ to IRN in the current topology, there is still some headroom for noise improvement. No appreciable noise contribution has been observed from the digital section of the TIA.

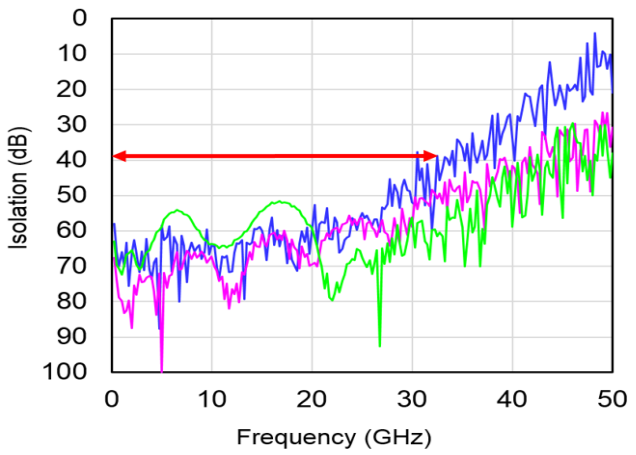

Fig. 7. Measured channel isolation between adjacent channels at Low (blue), medium (pink) and high gain (green) settings

Common mode rejection ratio $(\mathrm{CMRR})>40 \mathrm{~dB}$ was also measured as shown in Fig. 9, that enhances the immunity 
of differential TIA against any common mode disturbances. The DC current compensation circuitry can absorb up to $4 \mathrm{~mA}$ without any performance penalty.

The chip occupies an area of $2 \mathrm{~mm} \times 3.5 \mathrm{~mm}$. The power consumption is $\sim 300 \mathrm{~mW} /$ channel from a $3.3 \mathrm{~V}$ supply.

A detailed performance comparison of the presented quad channel TIA against the most relevant and recent publications is shown in Table 1 .

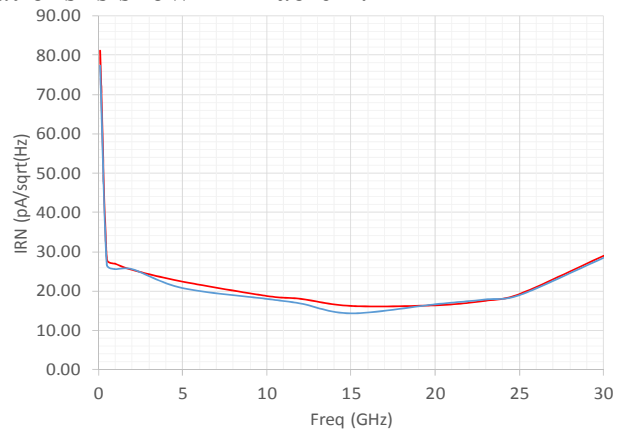

Fig. 8. Measured input referred noise of TIA at $\mathrm{Zt}$ of $7 \mathrm{~K} \Omega$ (blue) and $5 \mathrm{~K} \Omega$ (red)

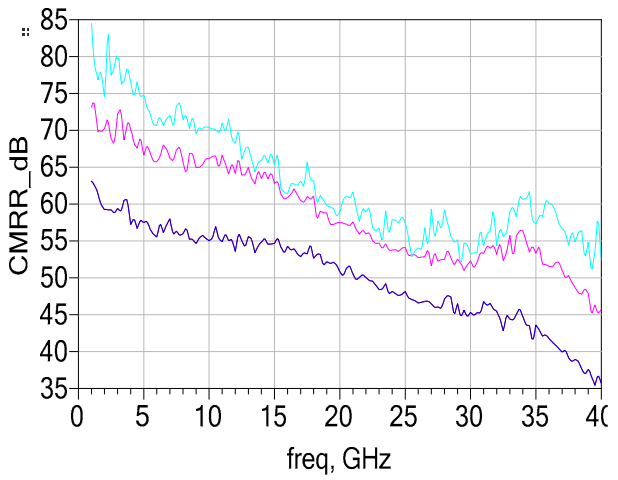

Fig. 9. Measured CMRR of TIA at low (blue), medium (pink) and high (sky blue) gain settings

Table 1: Comparison with previously published work

\begin{tabular}{|c|c|c|c|c|}
\hline & [2] & [3] & [4] & This work \\
\hline Publication & Jan-2016 & Feb-2013 & Mar-2008 & Not Published \\
\hline Technology & 0.13 um SiGe & 0.13 um SiGe & $90 \mathrm{~nm}$ CMOS & 0.13 um SiGe \\
\hline No. of Channels & 2 & 4 & 1 & 4 \\
\hline SPI or Analog Control & Analog & Analog & Analog & SPI and Analog \\
\hline Automatic Gain Control & Yes & No & Yes & Yes \\
\hline Data Rate $(\mathrm{Gb} / \mathrm{s})$ & 64 & 25 & 40 & $32 / 45$ \\
\hline \begin{tabular}{|l} 
Transimpedance, \\
differential $(\Omega)$
\end{tabular} & $10 \mathrm{~K}$ & $6.7 \mathrm{~K}$ & $2 \mathrm{~K}$ & $6.8 \mathrm{~K}$ \\
\hline Voltage THD (\%) & - & - & - & $\begin{array}{c}<0.9 \% \text { for Vout } \\
<500 \mathrm{mV} \text { for Input }< \\
2 \mathrm{mApp}\end{array}$ \\
\hline Voltage THD (\%) & $\begin{array}{l}<4.13 \text { for Vout } \\
<900 \mathrm{mV} \text { for } \\
\text { Input }<3 \mathrm{mApp}\end{array}$ & - & - & $\begin{array}{c}<2.5 \% \text { for Vout } \\
<900 \mathrm{mV} \text { for Input } \\
<3 \mathrm{mApp}\end{array}$ \\
\hline $\begin{array}{l}\text { Input referred noise density } \\
(\mathrm{pA} / \sqrt{ } \mathrm{Hz})\end{array}$ & 25 & 16 & 22 & 20 \\
\hline \begin{tabular}{|l}
$\begin{array}{l}\text { Power dissipation per } \\
\text { channel }(\mathrm{mW})\end{array}$ \\
\end{tabular} & 277 & 67.5 & 75 & 285 \\
\hline $\begin{array}{l}\begin{array}{l}\text { Maximum output vol tage } \\
(\mathrm{mV})\end{array} \\
\end{array}$ & 900 & 160 & 250 & 900 \\
\hline Die size $(\mathrm{mm} \times \mathrm{mm})$ & $\begin{array}{c}1.5 \times 1.3 \\
\text { (dual) }\end{array}$ & $\begin{array}{l}1.5 \times 3.3 \\
\text { (quad) }\end{array}$ & $\begin{array}{c}0.86 \times 0.65 \\
\text { (single) }\end{array}$ & $\begin{array}{c}2 \times 3.5 \\
\text { (quad+SPI) }\end{array}$ \\
\hline
\end{tabular}

\section{CONCLUSION}

This paper presented a highly integrated quad channel TIA with SPI and analog mode, combined dual interface and exhibits state-of-the-art linearity and isolation characteristics suitable for present and next generation micro-ICRs for coherent receiver applications. This TIA in a $\sim 2 \mathrm{~mm} \times 3.5 \mathrm{~mm}$ form factor, provides a value in terms of reducing size, complexity and cost of ICR module and paves the way for further integration.

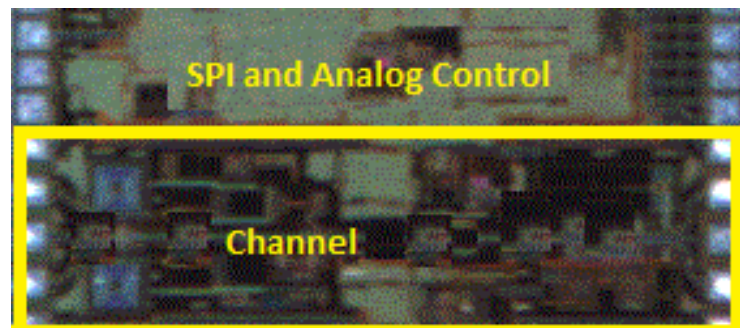

Fig. 10. Partial Chip Micro-Graph depicting SPI and RF channel layout outline

\section{ACKNOWLEDGMENT}

The authors would like to thank Oleh Krutko for design engineering support and Mike Tessaro for the specification guidelines. They would also like to acknowledge Youssef Boulette and Markus Wieczorek at Micram, Germany for design support. Many thanks to Bao Ellner, Jennifer Harris and Leszlie Beumer-Gray for layout support.

\section{REFERENCES}

[1] J. Zhang, et al. , "Compact Low-Power-Consumption 28Gbaud QPSK/16-QAM Integrated Silicon photonic/Electronic Coherent Receiver," IEEE 2016 International Solid-State Circuits Conference (ISSCC), pp 406407, Jan. 312016.

[2] Ahmed Awny, et al. , “23.5 A dual 64Gbaud 10k $\Omega$ 5\% THD linear differential transimpedance amplifier with automatic gain control in 0.13um BiCMOS technology for optical fiber coherent receivers," IEEE 2016 International Solid-State Circuits Conference (ISSCC), pp 406-407, Jan. 312016.

[3] G. Kalogerakis et al., "A quad $25 \mathrm{~Gb} / \mathrm{s} 270 \mathrm{~mW}$ TIA in 0.13 m BiCMOS with $0.15 \mathrm{~dB}$ crosstalk penalty," IEEE ISSCC Symp. Dig., pp. 116-117, Feb.2013.

[4] C. Liao et al., " $40 \mathrm{~Gb} / \mathrm{s}$ Transimpedance-AGC Amplifier and CDR Circuit for Broadband Data Receivers in $90 \mathrm{~nm}$ CMOS," IEEE J. Solid-State Circuits, vol. 43, no. 3, Mar. 2008.

[5] Behzad Razavi, "Design of Integrated Circuits for Optical Communications", Mc Graw Hill. 